\title{
Identifying Conceptual Differences Between Psychiatric Disorders and Neurological Disorders Although Both Are Disorders of Brain
}

\author{
Shrirang S. Bakhle \\ Dr. Bakhle Clinics, Mumbai, India
}

\begin{abstract}
With increasing understanding of neurobiological basis, Psychiatric Disorders are being considered Brain Disorders. As neurobiological basis of Psychiatric Disorders becomes known, some questions arise. Should all these disorders be called Neurological Disorders and be treated by Neurologists? If, both, Psychiatry and Neurology treat disorders of the same organ, brain, then, should departments of Psychiatry be merged with departments of Neurology? What are the defining features of Psychiatric Disorders as a group that differentiate them from Neurological Disorders? There is a need to define inclusion and exclusion criteria for distinguishing between Psychiatric and Neurological Disorders. There are practical needs for this. For example, what criteria guide the decision to admit a patient in Psychiatry Ward or Neurology Ward? This is also important from legal and stigma point of view. The theoretical need is nosological. What criteria determine whether a particular disorder should be included in Neurology or Psychiatry? The paper shows how it is impossible to differentiate between Psychiatric and Neurological Disorders on the basis of neuroanatomy or neurophysiology. The paper describes the vital importance of the term “mental” in Psychiatry (e.g., "Mental” Disorders, "Mental” Status Examination, etc.) and how it cannot be replaced by "Neurological” or "Brain". The paper discusses how Psychiatric Disorders are basically "Mental” Disorders (disorders of mind). It shows how mind is a real and specific entity. It gives a novel definition of mind using a specific concept of "functional organization". The article gives a precise description of mind-brain relationship using the concepts of physical parts, functional parts and active functional organization. This description answers many questions such as "Why no biomarkers have been found for any of the Mental Disorders" and the topic in the title. The article also explains how this concept of mind provides a better framework for describing the mental events in normal persons and in Mental Disorders-as compared to the concepts of Cognition or Higher Functions or Executive Functions. It shows the fallacy of using the term "Behavioral” Disorders. The article shows how there is no other answer to the question of "What is the difference between Psychiatric and Neurological Disorders?”
\end{abstract}

Keywords: behavioral disorders, cognition, DSM-5, functional organization, mental disorder, mind, mind-brain

With increasing understanding of neurobiological basis, Psychiatric Disorders are being considered Brain Disorders. As neurobiological basis of Psychiatric Disorders becomes known, some questions arise. Should all these disorders be called Neurological Disorders and be treated by Neurologists? If, both, Psychiatry and

Shrirang S. Bakhle, M.B., Director, Dr. Bakhle Clinics. 
Neurology treat disorders of the same organ, brain, then should departments of Psychiatry be merged with departments of Neurology?

What are the defining features of Psychiatric Disorders as a group that differentiates them from Neurological Disorders?

There is a need to define inclusion and exclusion criteria for distinguishing between Psychiatric and Neurological Disorders. There are practical needs for this. For example, what criteria guide the decision to admit a patient in Psychiatry Ward or Neurology Ward? This is also important from legal and stigma point of view.

The theoretical need is nosological. What criteria determine whether a particular disorder should be included in Neurology or Psychiatry?

Intuitively everyone understands the difference. But as a scientific method, it is important to precisely describe the specific differentiating criteria that distinguish Psychiatric disorders from Neurological disorders although both the disorders happen in the brain. It is relatively easy to say that Neurological Disorders are disorders of the brain (and the nervous system). But it is more difficult to pinpoint the defining and differentiating features of Psychiatric disorders as a group.

Answering these questions is also important to establish the unique specialty of Psychiatry and to answer some of the objections of the Anti-Psychiatry proponents, who have called Psychiatry a pseudoscience and Psychiatric Disorders a myth (Torrey, 1975; Szasz, 1960; Weitz, 2016; Ross \& Pam, 1995; Nasrallah, 2011). No one says that Neurological Disorders are a myth. So, what are the reasons behind people saying that about Psychiatric disorders? Thus answering this question is vital to establish the unique identity of Psychiatry and Psychiatric Disorders.

This is not merely an exercise to divide the turf for Psychiatrists and Neurologists by randomly allocating disorders. It is a fundamental effort to identify the conceptual differences between these two sciences.

With advancing understanding of neurobiological basis of Psychiatric Disorders, it can no longer be said that Psychiatric Disorders are "functional" and Neurological Disorders are "structural" or "organic". But on the other hand, just because neurological basis has been found does not mean that all Psychiatric Disorders should be called Neurological Disorders and that all psychiatric patients should be treated by Neurologists.

Psychiatric Disorders and Neurological Disorders cannot be differentiated on the basis of neuroanatomy or neurophysiology. Can it be said that disorders of some parts of brain (such as hippocampus) are Psychiatric Disorders and disorders of other parts are Neurological? The answer is negative. Or, can it be said that disorders of Dopaminergic system are Psychiatric Disorders and disorders of other systems are Neurological Disorders? Again, the answer is negative.

To say that there is a specialty of Neuropsychiatry is bypassing the question, not answering it. When it is said that Neuropsychiatry is a merger of the two sciences, it is acknowledged that these are two distinct sciences. There is a conceptual difference between Psychiatry and Neurology, and this paper seeks to present it.

\section{Different Viewpoints about the Differences Between Psychiatric Disorders and Neurological Disorders}

There are a variety of positions regarding this issue.

In ICD-10, the chapters from III to XIV are dedicated to different physiological systems (such as Circulatory system or Respiratory system) or to different anatomical parts (such as eye or ear). Therefore, it is 
important to note that the ICD-10 has assigned Mental Disorders and Neurological Disorders to separate chapters (V and VI). It underlines the important conceptual distinction between these two categories of disorders even though both are related to the brain (ICD-10, 2010). Even in the draft of the forthcoming ICD-11, Mental Disorders and Neurological Disorders have been assigned to separate chapters (ICD-11 Beta Draft, 2016).

Kaplan and Sadock’s Comprehensive Textbook of Psychiatry, 9th Ed., (Michels, 2009) says the following about this issue: "There is much greater difference in the skill sets of neurologists and psychiatrists than in their scientific knowledge bases, and there are certainly more than enough patients for both. We can look forward to a healthy continued debate about where best to draw the boundary...” Two important points can be noted from this. First, there is a boundary between Neurology and Psychiatry. So, these two are distinct sciences. Secondly, Neurologists and Psychiatrists require different skill sets even if they both deal with the brain.

This concept is echoed by Ronald Pies in his article "Why Psychiatry and Neurology cannot simply merge" (Pies, 2005). He describes how the discourses and terms used in Psychiatry and Neurology are different.

On the other hand, there are many who wish or predict that Psychiatry and Neurology will eventually merge. For example, White, Rickards and Zeman, in their article, "Time to end the distinction between mental and neurological illnesses", say the following: "Yet the dominant classifications of mental disorder-the International Classification of Diseases (ICD) and the Diagnostic and Statistical Manual (DSM) - continue to draw a sharp distinction between disorders of the mind, the province of psychiatry, and disorders of brain, the province of neurology." The article expresses a wish that both "the conditions should be grouped together as disorders of the nervous system” (White, Rickards, \& Zeman, 2012).

The proponents of the Anti-Psychiatry movement also recommend that only Neurology should remain. But they say this from a different perspective. According to them, the concept of mental disorders is a myth. There are either brain disorders or there are difficulties in social adaptation. Hence the "pseudoscience" of Psychiatry should be abolished.

The Stanford Encyclopedia of Philosophy, in the article "Mental Illness" predicts, "It is likely that as neuroscience progresses, the domains of neurology and psychiatry will start to merge” (Perring \& Zalta, 2010).

\section{Psychiatric Disorders Are “Mental” Disorders}

The word "mental" is ubiquitous in Psychiatry: "Mental" Status Examination (MSE), DSM-5 is the DSM of "Mental" Disorders, National Institute of "Mental" Health and so on. Will it be appropriate to substitute "mental” with, say, "brain” or "neurological”? Will it be correct to say, "Brain” Status Examination or National Institute of "Neurological" Health? Will it mean the same?

The DSM- IV-TR said the following about this issue: “... unfortunately the term (mental) persists in the title of DSM-IV-TR because we have not found an appropriate substitute” (DSM-IV-TR, 2000). Thus, in spite of long deliberations, they could not replace the term "mental" with other terms such as "brain" or "neurological". And the DSM-5 continues to carry the term "Mental" in its title: "Diagnostic and Statistical Manual of Mental Disorders", 5th Ed (DSM-5, 2013).

Thus Psychiatric Disorders are basically "mental” disorders.

\section{What Is the Meaning of “Mental”?}

It is interesting to note that such an important term is not even mentioned or defined in Psychiatry or Psychology dictionaries such as Campbell's Psychiatric Dictionary (Oxford, 2009), Oxford Dictionary of 
Psychology (Colman, 2009), American Psychological Association’s Online Glossary of Psychological Terms (accessed 2016).

According to the Oxford English Dictionary (2010), “mental” means “1. Relating to the mind; 2. Relating to disorders or illnesses of the mind".

So can it be said that Psychiatric ("Mental") Disorders are disorders of the mind and Neurological Disorders are disorders of the brain? Is "mind" the unique identifying and differentiating feature of Psychiatry and Psychiatric Disorders?

To understand this point, there is a need to understand the inclusion criteria for determining that a particular disorder is a "Mental" Disorder. And what are the exclusion criteria that can used to determine that a particular disorder is not a "Mental" Disorder but is a Neurological Disorder?

\section{Understanding the Inclusion and Exclusion Criteria of the DSM-5}

The Diagnostic and Statistical Manual of Mental Disorders, 5th Ed. (DSM-5) is a comprehensive compendium of "Mental" (Psychiatric) Disorders. This means that every "Mental" (Psychiatric) Disorder is included in the DSM-5. It also means that no non-mental disorder is included in the DSM-5. Hence Neurological Disorders are not included in the DSM-5.

So by studying the inclusion and exclusion criteria of DSM-5, it will be possible to understand the identifying and differentiating features of Mental (Psychiatric) Disorders as compared to Neurological Disorders.

Unfortunately, the DSM-5 does not describe any inclusion or exclusion criteria for a disorder to be included in DSM-5 or to be excluded from it.

But an attempt can be made to understand these criteria by studying some disorders that have been included and some disorders that have been excluded.

For this, three disorders can be examined: (1) Conversion Disorder with Paralysis; (2) Hemiplegia due to a vascular event (for example, Hemiplegia due to Cerebral Infarct); and (3) Vascular Neurocognitive Disorder.

The Conversion Disorder and Vascular Neurocognitive Disorder are considered "Mental" Disorders and hence are included in the DSM-5. Hemiplegia is excluded from the DSM-5 as it considered a Neurological Disorder and not a Mental Disorder. This is in spite of the fact that all these disorders are related to the brain.

So what are the features that differentiate these two groups?

(1) Clinical features:

Both, Conversion Disorder with Paralysis as well as Hemiplegia, have paralysis as the presenting feature. Hence this is not a differentiating feature between them.

(2) Vascular etiology:

Both, Hemiplegia and Vascular Neurocognitive Disorder, have vascular etiology. Hence this is not a differentiating feature.

(3) Dysfunctions in mind:

Both Conversion Disorder and Vascular Neurocognitive Disorder have "mental" dysfunctions, i.e., dysfunctions in the mind. And therefore, they are considered as "Mental" Disorders and are included in DSM-5. But Hemiplegia does not have any dysfunction in the mind and hence is excluded from the DSM-5.

Every "Mental" Disorder has dysfunctions in the mind-with or without demonstrable dysfunctions in the brain. Are there any disorders in the DSM-5 that do not have "mental" dysfunctions (dysfunctions in the mind of the patient)? The answer is negative. 
Thus "mind" and "dysfunctions in the mind" are the unique identifying and differentiating features of Psychiatry and "Mental” (Psychiatric) Disorders.

\section{“Mind and Brain! Here We Go Again!”}

Undoubtedly, the moment the terms mind and brain are used, a set of questions arises: What is mind? Is it different from brain? What is the relationship between mind and brain? Are "Mental" Disorders, disorders of mind or brain or both?

Also, why should the seemingly old fashioned term "mind" be used? Why not use the more modern scientific terms like "Cognition" or "Higher Functions" or "Executive Functions"? And, is it correct to use the term "Behavioral” Disorders instead of "Mental” Disorders?

\section{Mind: The Central Key Piece of this Entire Puzzle}

The mysterious nature of the mind and its relationship with the brain has been a philosophical conundrum for millennia. Psychiatry is the medical science that evolved to treat "mental" disorders, i.e., the disorders of the mind. The NIH's US National Library of Medicine Website describes the "Diseases of the Mind: Highlights of American Psychiatry through 1900”. It says: "Benjamin Rush, often called 'The father of American Psychiatry', wrote the first systematic textbook on mental diseases in America entitled Medical Inquiries and Observations upon Diseases of the Mind published in Philadelphia in 1812" (accessed 2016).

Please note how the US NLM's webpage that describes the history of American "Psychiatry" is titled "Diseases of the 'Mind'". And the Father of American "Psychiatry" wrote the first textbook of "mental" diseases describing the diseases of the "mind".

But the inability to define and describe the mind has resulted in the all the confusions in Psychiatry such as the dilemma of the DSM-IV-TR (described above) and the challenges of the Anti-Psychiatry movement who have called Psychiatric Disorders a myth. This inability has also resulted in Psychiatry dumping the enigmatic mind in favour of the more concrete brain in search of a solid basis for Psychiatry and Mental Disorders. For example, see the direction of the Research Domain Criteria Project (RDoC Project) of National Institute of Mental Health (Cuthbert, 2013).

But the shift of focus from mind to brain has resulted in the fundamental unanswerable question: What is the difference between Psychiatric Disorders and Neurological Disorders if both are disorders of the brain? If the Psychiatric Disorders are disorders of the brain, then what about Neurological Disorders? They, too, are disorders of the brain. So what is the difference between the two? This and the other questions can be answered only by acknowledging that "Mental" Disorders are primarily disorders of the mind. There is no other answer to this question.

But to solve this confusion, there is a need for a precise definition and description of mind.

\section{What Is “Mind”?}

The humans are conscious, intelligent creatures. They are aware of different sensations, beliefs, wishes, emotions present in the mind. The metacognition occurs in the mind. The perception of environmental events also occurs in the mind.

Humans can consciously analyze every event: what has happened, why it has happened, what could have happened, what is likely to happen in future, etc. As a result of this analysis or processing, people experience 
emotions in the mind: happiness, sadness, fear, anger. Then based on the wishes, people take conscious decisions in the minds and do purposeful, deliberate actions to fulfill the wishes.

All this happens in the mind.

"Mind is the aggregate of everything that a person is aware of".

Thus mind is the aggregate of the beliefs, wishes, emotions, the attention, the mental abilities and all the mental events.

\section{Mind Is an “Active Functional Organization” and Not Just a Random Collection of all These Functional Parts}

Mind is a specific functional organization of all these specific interactive parts. It is not just a random list of all these. An "active functional organization" is capable of receiving specific inputs, processing them to produce specific outputs. Thus it is capable of doing work which is more than the sum of work that its individual parts can individually do. All the parts function cohesively in a unified manner to do the processing. This enables each person to process different inputs to produce different outputs consciously and purposefully. If a human can consciously do any thinking or voluntary activities, it is the result of this "active functional organization" called mind.

\section{Is Mind the Same as Brain? What Is the Relationship Between Mind and Brain?}

Undoubtedly, the mind is created in the brain. But the mind is not the same as brain.

First, there is a category difference between the two: People are aware of the mind and its contents. But they are not aware of brain, neurons, etc.

Secondly, there is a function-structure relationship between mind and brain. The parts of the mind are functional parts: beliefs, wishes, emotions and all the mental events (events in the mind that people are aware of). The parts of brain are the physical or structural parts. When brain parts perform their functions, they produce the functional parts of the mind. These interactive functional parts lead to the formation of the active functional organization called mind. This is a very crucial concept to understand.

In order to illustrate this concept of "functional parts, physical parts and functional organization", the example of another functional organization, a school, can be studied. The building, the people and the children are the physical parts. The Dean, teachers, students and classrooms are the functional parts. Here, can it be said that the physical parts are different from functional parts? When an individual physical part performs its function, that function becomes a functional part. For example, when a man becomes a teacher or a child becomes a student. The interactions between the functional parts, lead to the formation of the "functional organization". Then this functional organization becomes capable of processing complex inputs to produce complex outputs. In this case, the output is educating the children.

For this functional organization, specific physical parts and functional parts are required. For example, if people and children are randomly picked up from the street and put in any randomly picked building, it will not become the active functional organization called school. It will not be able to process the inputs to produce the specific output.

On the other hand, consider the turnover in a school. The teachers retire or leave and new teachers join. Students pass out and new students join. The building may change. Yet the functional organization remains—-performing its function of processing inputs to produce outputs. Thus a "functional organization" is a 
well-defined real entity although there may not be a strict one to one correlation between physical parts and functional parts.

The mind is a similar active functional organization of functional parts that are produced in the physical parts of the brain.

\section{The Importance of Understanding the Difference Between Physical Parts, Functional Parts and the Functional Organization}

When a school is photographed, what exactly is being photographed? Is the functional organization called school being photographed or are only the physical parts that generate the functional parts are being photographed? Is it possible to photograph the education that is being created in the minds of the students by taking photo with a camera? The answer is negative. Here the ontological difference between the physical parts and the functional parts comes into the picture.

This point has tremendous relevance to the discussion about the mind-brain relationship. When an fMRI or a PET scan is done, what is being photographed? Are the mind parts (functional parts) being photographed or brain parts (physical parts) being photographed? Clearly, the brain parts are being photographed. At the most, it can be said that the scans are photographing the brain parts while they are producing the functional mind parts. It is impossible to photograph the functional mind parts such as beliefs, wishes or emotions with an fMRI. Can an "fMRI Report" be like this: "The subject was thinking angrily about the office colleague, John, who had insulted him yesterday in front of the office staff. The subject was thinking about when and how to take revenge"? Such an fMRI report would mean photographing the mind. This is impossible in the present or the near future. It is important to note this point when the mind and brain is being studied.

\section{The Properties of a "Functional Organization"}

It is important to understand this concept in more detail in order to understand the enigmatic nature of the mind. A "functional organization" is formed when individual physical parts are aligned in a particular manner. Each individual physical part is capable of performing a small function depending on its properties. When these aligned individual physical parts perform their functions in a definite temporal sequence, the result is the formation of a functional organization. The net resulting function of this functional organization is far greater than the mere sum of the functions of each individual part.

The example of another functional organization, a robot, can be studied to understand this point.

Consider all the parts of a robot lying in a heap. All the parts are exactly the same as present in a functional robot. If the physical properties such as weight or volume are measured, there will be no difference between the parts of the robot lying in a heap or the parts in a functional robot. Even when the parts are kept separately, each individual part is capable of doing a small job that it is made for. Yet, when the same parts are aligned as a "functional organization", they can do work which is far bigger than the sum of the works that they can do individually. In the example of the robot, the different motors individually do simple work: rotate the axles. But when they are arranged as a part of a "functional organization", they, together with other parts, can effect highly complex robotic movements.

There are plenty of examples of functional organizations. Each biological cell is a functional organization of specific physical parts. The physical parts are aligned in such a way that the interactions of their functional 
parts lead to the formation of an active functional organization called a living cell. This functional organization is capable of receiving and processing complex inputs to produce complex outputs. Different organs or living creatures, too, are examples of functional organizations. Schools, companies or governments are some examples of functional organizations.

Thus it is important to recognize an entity called "active functional organization".

A "functional organization" is a real entity but it does not have a separate material existence that can be measured in physics terms like weight or volume. It is a pattern created in an alignment of physical parts. Its existence can be understood by observing its ability to process complex inputs to produce complex outputs. If the existence of a functional organization like government can be accepted, the existence of the active functional organization called mind can also be accepted.

If a human can consciously do any thinking or voluntary activities, it is the result of this active functional organization called mind.

\section{Mind and the "Mental" Disorders}

The "mental" disorders are disorders of this functional organization called mind. Each "mental" disorder involves dysfunctions in one or more of the functional parts of mind. Sometimes they may involve demonstrable dysfunctions in the physical parts of the brain but not always. As seen earlier, investigations such as fMRI or PET scan cannot photograph the functional mind parts. Hence they cannot photograph the dysfunctions in the functional mind parts that are seen in the mental disorders. At best they can photograph the physical brain parts while they are producing the dysfunctions in the functional parts of the mind.

It is important to note that there is no strict one to one correlation between physical brain parts and functional mind parts as well as in the dysfunctions in both of these. Hence it is not possible to diagnose dysfunctions in functional mind parts using investigations like fMRI or PET scan that can photograph the physical brain parts. This point clarifies the reason why no biomarkers (based on brain investigations) have been found for any of the mental disorders. At the most, these investigations can be used in the other direction: to find out the brain part correlates of the functional mind parts and their dysfunctions.

Yet it is important to acknowledge the reality of the active functional organization called mind as well as the dysfunctions in the functional parts of the mind seen in "mental" disorders.

\section{Are "Mental” Disorders, Disorders of Mind or Brain?}

Since mind is created in the brain, is it correct to say that "mental" disorders are disorders of the brain? The brain performs many different functions. Producing mind is one of them. Thus mind is one subset of the functions produced by the physical parts of the brain.

Is it appropriate to say that a Department of Psychiatry is the same as the Medical College in which it is housed? By pointing to the Department of Psychiatry (DoP), it can be said that "This is DoP" or it can be said that "This is Medical College". Both the times it will be correct. Yet, it is easy to know that the DoP is not the same as the Medical College. Similarly, it can be said that "Mental Disorders are disorders of mind" or it can be said that "Mental disorders are disorders of brain". Both the descriptions are right. But the description that "Mental Disorders are disorders of the mind", is scientifically precise. The original topic of this article needs to be seen in this light. 


\section{What Is the Difference Between Psychiatric Disorders and Neurological Disorders If Both are Disorders of the Brain?}

It can be said that the Psychiatric (or Mental) Disorders are disorders of the brain. But the description that the Psychiatric (or Mental) Disorders are disorders of the mind, is scientifically precise.

Similarly, it can be said that Psychiatry is study of disorders of the brain. But the description that Psychiatry is study of disorders of the mind, is scientifically precise.

It is important to note that the statement, "Psychiatric Disorders are disorders of the mind", does not contradict the fact that Psychiatric Disorders happen in the brain.

\section{After the Clinical Examination of a Patient, Does a Psychiatrist Conclude “This Patient Has Dysfunction in Ventral Tegmental Area”?}

After the clinical examination, does a Psychiatrist diagnose dysfunctions in different parts of the brain such as Ventral Tegmental Area or Nucleus Accumbens? The Psychiatrist may conclude that this patient is suffering from "intense sadness and mild fear related to the topic of failure in exam". These are dysfunctions in parts of the mind. The Psychiatrists may use different methods of treatment (such as Pharmacotherapy or Brain Stimulation Therapies) that involve modifying various aspects of brain. But the goal of Psychiatrists is to diagnose and treat the dysfunctions in the mind.

\section{Why Use an Old Fashioned Term like "Mind"? Why not Use the More Modern Terms like “Cognition”, “Executive Functions” or "Higher Functions”?}

There are two distinct advantages of using the term mind as compared to the use of the other terms. Firstly, mind is a more comprehensive concept as compared to the other terms. And secondly, the term "mind" denotes an active functional organization of interactive parts capable of receiving inputs and processing them to produce outputs. The other terms are merely lists of functions.

For example, consider the term "cognition". The Oxford Dictionary of Psychology defines cognition as "the mental activities involved in acquiring and processing information" (Colman, 2009). Obviously, the emotions are not included in the concept of cognition. The textbook "Cognition” by Ashcraft and Radvansky, is an example of this. It defines "Cognitive science" as: "the scientific study of thought, language, the brain —in short, the scientific study of mind". As can be seen, the emotions are missing in this definition. Not only that, but in this entire textbook of 477 pages, just one and half page is devoted to the description of emotions (Ashcraft \& Radvansky, 2010).

The tremendous importance of emotions in mind and mental disorders as well as the intense interplay between cognition and emotions is well known. By excluding emotions, the term cognition becomes an incomplete account of the mental events in normal persons and in persons with "mental" disorders. In comparison, the mind is a complete and comprehensive term when the mind is defined as the aggregate of everything that a person is aware of: sensations, beliefs, wishes, emotions, attention, mental abilities as well as the mental events.

The terms Executive Functions (EF) or Higher Functions (HF) are even narrower. Both are merely lists of a few functions. As compared to the entire scope of the mind (as the aggregate of everything that a person is aware of), the EF or HF represent only very few of the functions that the human mind is capable of. Also, the mind represents an "active functional organization" as compared to EF or HF which are just lists. So if a 
comprehensive framework to describe the mental events (in normal persons as well as in persons with Mental Disorders) is required, the mind is a much better option as compared to EF or HF.

\section{Is It Correct to Use the Term “Behavioral Disorders” Instead of “Mental Disorders”?}

“Introduction to Psychology” by Morgan, King, Weisz and Schopler defines behavior: "Behavior includes everything a person or animal does that can be observed in some way" (Morgan, King, Weisz, \& Schopler, 2001). So, can any "altered behaviour" be termed as "Behavioral Disorder"? Consider orthopedic problems. They, too, lead to markedly altered or abnormal "behavior". For example, abnormal facial expressions, movements, gait, bladder and bowel habits as well as functionality. Can all such disorders be called as Behavioral Disorders? The answer is negative.

A disorder is called a Behavioral Disorder when the dysfunction is in the mind with resultant behavioral manifestations. So, Behavioral Disorders are actually disorders of the mind. If the Motor Pathway is studied, it easy to understand how the behavior is the output from the mind. Behavior is merely the externally "observable" output of the mind.

Thus Psychiatric or "Mental" Disorders are best described as disorders of the mind. Terming them as Behavioral Disorders is incorrect.

\section{Conclusion}

Mind is a subset of the functions of the brain. But mind is not the same as brain.

The Psychiatric Disorders are "Mental" disorders. This means that they are primarily disorders of the mind. If "Mental" Disorders are defined as disorders of the brain, it is correct. But, if the "Mental" Disorders are defined as disorders of the mind, it is being scientifically precise. The statement, "Psychiatric Disorders are disorders of the mind" does not contradict the fact that Psychiatric Disorders happen in the brain.

Similarly, if Psychiatry is defined the study of disorders of the brain, it is correct. But if Psychiatry is defined as the study of disorders of the mind, it is being scientifically precise.

Thus, Psychiatry should be defined as the study of Mental Disorders which means disorders of the mind.

Neurology is a broad term which means study of disorders of the brain (and nervous system).

\section{References}

Ashcraft, M. S., \& Radvansky, G. A. (2010). Cognition (p. 2). Dorling Kindersley: Pearson Education Inc..

Campbell, R. J. (2009). Campbell's Psychiatric Dictionary (9th Ed.). New York, N.Y.: Oxford University Press.

Colman, A. M. (2009). Oxford Dictionary of Psychology (3rd Ed., p. 143). Oxford UK: Oxford University Press.

Cuthbert, B. N., \& Insel, T. R. (2013). Toward the future of psychiatric diagnosis: The seven pillars of RDoC. BMC Medicine, 11, 126. doi: $10.1186 / 1741-7015-11-126$

International Classification of Diseases and Related Health Problems (10th Revision) (ICD-10). (2016). World Health Organization. Retrieved April 2, 2016, from http://apps.who.int/classifications/icd10/browse/2016/en

International Classification of Diseases and Related health Problems (11th Revision-Beta Draft) (ICD 11-Beta Draft) Retrieved April 1, 2016, from http://apps.who.int/classifications/icd11/browse

Michels, R. (2009). Kaplan and Sadock's Comprehensive Textbook of Psychiatry (9th Ed., p. lvi). Philadelphia, P.A.: Wolters Kluwer/ Lippincott Williams and Wilkins.

Morgan, C. T., King, R. A., Weisz, J. R., \& Schopler, J. (2001). Introduction to psychology (7th Ed., p. 6). Mcgraw-Hill Inc.: Tata McGraw-Hill.

Nasrallah, H. A. (December 2011). The antipsychiatry movement: Who and Why. Current Psychiatry, 10(12). 
Ozarin, L., \& North, M. (Ed.). (2013, September 17). Diseases of the mind: Highlights of American psychiatry through 1900-Benjamin Rush. U.S. National Library of Medicine. Retrieved April 2, 2016, from http://www.nlm.nih.gov/hmd/diseases/benjamin.html

Perring, C., \& Zalta, E. N. (Ed.). Mental illness: The Stanford Encyclopedia of Philosophy (Spring 2010 Edition). Retrieved April 2, 2016, http://plato.stanford.edu/archives/spr2010/entries/mental-illness/

Pies, R. (Summer, 2005). Why psychiatry and neurology cannot merge. Journal of Neuropsychiatry and Clinical Neurosciences, 17, 304-309.

Ross, C. A., \& Pam, A. (1995). Psudoscience in biological psychiatry. New York, N.Y.: John Wiley and Sons, Inc..

Soanes, C. (Ed.). In S. Hawker, \& J. Elliott (2010). Oxford English Dictionary (26th impression). Oxford University Press.

Szasz, T. (1960). The myth of mental illness. New York: Hoeber-Harper.

The Diagnostic and Statistical Manual of Mental Disorders, 5th Ed., (DSM-5). (2013). Washington, D.C.: American Psychiatric Association.

The Diagnostic and Statistical Manual of Mental Disorders, 4th Ed., Text Revision, p. xxx, (DSM-IV-TR). (2000). Washington, D.C.: American Psychiatric Association.

Torrey, E. F. (1975). The death of psychiatry. New York, N.Y.: Penguin Books.

Weitz, D. (1998). 25 Good reasons why psychiatry must be abolished. Retrieved April 1, 2016, from http://www.antipsychiatry.org/25reason.htm

White, P. D., Rickards, H., \& Zeman, A. Z. J. (2012). Time to end the distinction between mental and neurological illnesses. BMJ, 344, e3454. 